\title{
Dynamic Smooth Handover Method based on Transition Zones
}

\author{
Ren Wang ${ }^{1,2, a}$, Yi Wang ${ }^{1,2, b, *}$, Liangliang Tian ${ }^{2, c}$ and Yan Liu ${ }^{2, d}$ \\ ${ }^{1}$ Key Laboratory of Intelligent Computing \& Signal Processing, Ministry of Education, Anhui \\ University, Hefei, China \\ ${ }^{2}$ School of Electronics and Information Engineering, Anhui University, Hefei, China \\ a952175687@qq.com, bmesh_wy@163.com, c1364485545@qq.com, ${ }^{\mathrm{d}}$ 2925480218@qq.com \\ *corresponding author
}

Keywords: CRE, Transition zone, Ping-Pong effect

\begin{abstract}
In order to improve coverage and load balancing in cellular networks, CRE (Cell Range Expansion) which is introduced in LTE-Advanced has drawn significant attentions. However, the ping-pong effect resulting from cell handover is still a challenge. In this paper, we propose a new dynamic smooth handover method based on transition zones for the celledge users to deal with the ping-pong effect. The cells' boundaries extend to transition zones within which the users do not switch serving cells. On the other hand, the range of the transition zones can be adjusted depending on performance requirements. Simulation results show that our proposed method is near optimum throughput and traffic load performance without the ping-pong effect.
\end{abstract}

\section{Introduction}

With the increase of mobile terminals and the emergence of various new applications, network traffic load increases exponentially, and high data rate and high quality service are required in cellular networks $[1,2]$. Heterogeneous networks constituted of macro cell base stations and lowpower nodes (such as pico, femto and relay nodes) will be promising to improve the network capacity, which have drawn more and more attentions $[3,4]$.

Heterogeneous networks can improve coverage and throughput of next-generation mobile communication systems, but they also present some problems [5]. For example, low power nodes and macro base stations use the same frequency resource and cause strong interference. In addition, according to traditional methods, users will choose serving stations based on the maximum RSRP (Reference Signal Received Power), and most of the users may establish connections with macrocells. Thus, the cooperative mode of the low power nodes is no longer significant. CRE (Cell Range Expansion) is introduced by adding an appropriate bias to the pilot signal of a picocell, forcing more users to choose picocells [6]. Although it will lead to the decrease of the network throughput, the load balancing between macrocells and picocells can be achieved. Bias value will directly affect the overall network status. The DL (DownLink) RSRP of the pico users (User Equipments) located in the expanded range will be low and the SINR (Signal to Interference plus 
Noise Ratio) decreased seriously when the bias is too big. The macro users that caused UL (Uplink) interference cannot link to small sites. And the load of a macro base station will be still high when the bias is too small [7].

Users can select the access TPs (Transmission Points) every minimum TTI (Transmission Time Interval) which is usually equal to $1 \mathrm{~ms}$ [8]. In addition to throughput, there are more options available to choose for UEs (User Equipments) in heterogeneous networks. However, handover control scheme have to ensure that normal communication will not be affected. Communication system performance will be reduced by inappropriate cell selection. Though CRE is introduced, one major problem of ping-pong effect remains and even causes the interruption of communication. Typically, cell handover can be divided into two classes: horizontal and vertical. Horizontal handover occurs between the traditional homogenous networks, while vertical handover which becomes a research hotspot focuses on the heterogeneous networks. Vertical handover is a rather complicated process, which requires low latency, low power consumption, and occupys as little bandwidth as possible. It puts forward high requirements for broadband wireless access technique, signal detection, channel allocation and optimization for radio resource distribution. If the signal strength of two base stations in certain areas dramatically change, the user will switch back and forth between the two base stations, causing ping-pong effect.

Cell handover and re-selection are major concerns for mobile communications. In this paper, we propose a new smooth handover method based on transition zones for the users located in the celledge to solve the ping-pong effect. Unlike hysteresis margin, the given method is founded on the range expansion. On the other hand, the method can effectively tradeoff the system throughput and the traffic load.

The remainder of the paper is organized as follows. In section 2 we present our system model. And our proposed method is introduced in section 3. Section 4 presents simulation results. Finally, conclusions are given in Section 5.

\section{System Model}

\section{A. Heterogeneous networks}

As is shown in Figure 1, according to the 3GPP detailed description of the deployment on heterogeneous networks, we consider the coexistence of macro and pico sites in heterogeneous networks. In order to describe our proposed method better, the simplified model just contains a macrocell and several picocells. Users share the same time-frequency domain resources. MUE (Macro User Equipment) establishes a connection with the macrocell, and PUE (Pico User Equipment) establishes a connection with a picocell. The solid lines represent the current connection, and the dotted line represents the potential connection. Users move randomly at a unified rate of $0-3 \mathrm{~km} / \mathrm{h}$.

B. Cell range expansion

Cross-tier interference and imbalance of uplink and downlink will exist simultaneously in heterogeneous networks with many small sites. Small base stations, due to their low transmitting power, result in less access load. CRE is an available solution to achieve load balancing, for some users are forced to access low power base stations through optimizing the bias value. And our proposed method is based on range expansion.

C. Transition zone

In order to deal with the ping-pong effect and improve system performance, the transition zone is given in this paper. As is shown in Figure 1, the solid line identifying cells' boundaries in the traditional CRE is extended to an area. If ping-pong effect is not effectively addressed, users along 
the cells' boundary will switch back and forth between base stations. Therefore, we propose the transition zone to improve the efficiency and reduce the communication overhead.

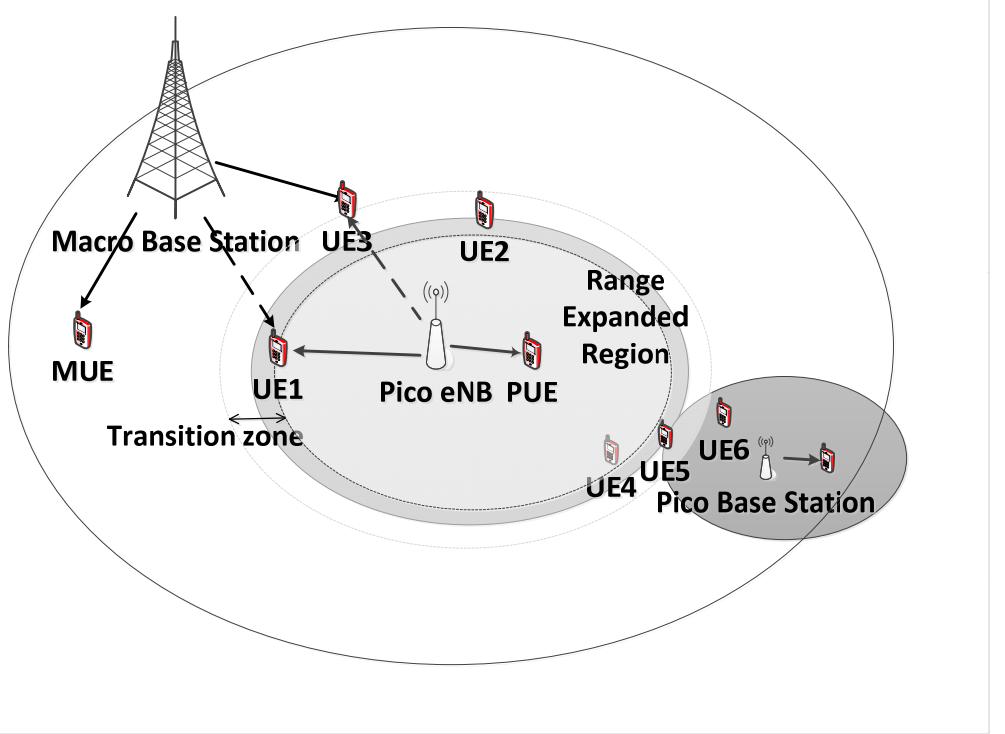

Figure 1 System model

\section{Proposed Method}

The method is presented in this section. In homogeneous networks, users choose serving cells providing maximum RSRP which are usually neighbouring cells. It can be denoted as

$$
C E L L \_s e r v i n g=\arg \max \left\{R S R P \_i\right\}
$$

where $i$ denotes the $i$-th neighboring base stations.

However, if this decision scheme is applied directly in heterogeneous networks, it probably leads to most of users selecting the macrocell as their serving cell. It is proposed by CRE that we can add a bias value to RSRP to drive more users to select picocells as their serving cells. And the operation is given as

$$
C E L L \_s e r v i n g=\arg \max \left\{R S R P \_i+b i a s \_p \_i\right\}
$$

where

bias_p_ $i=0$, if $i$ is a macro base station,

bias_p_i>0, otherwise.

By adding an optimal bias value, users can be forced to access to the picocells and load balancing can be achieved. But there is still an overlapped area between extended picocells' coverage and macrocells' coverage. To deal with ping-pang effect, two boundaries of the transition zone, bias_a and bias_b, can be given by

$$
\text { bias_a<bias_p<bias_b }
$$

As depicted in Figure 2, the users' choices of service station are made during the move. The location and track of the user will determine which the serving cell is. The corresponding steps are as follows:

If a user moves towards the macro base station and reaches the boundary of bias_a, handover is not allowed. And the communication link is maintained when the user enters the transition zone. 
Next, if the user in the transition zone returns to the small site's coverage, no handover is to happen. Unless the user reaches the boundary of bias_b and cross the transition zone, cell handover is initiated.

Then, if the user returns to the transitional zone through the boundary of bias_b, the macrocell still be the serving cell. Similarly, if the user crosses the transition zone, the small site is selected.

As depicted in Figure 3, bias_a and bias_b change along with the network environment. And the two parameters are in a reasonable range. The achievable sum rate of users in the transition zone served by small base stations is $R p$, and that served by macro sites is $R m$. Changes in boundaries of bias_a and bias_b can be described as follows:

Judge that whether the difference between $R p$ and $R m$ is greater than a threshold value.

If the judgment result is YES, suitable serving cell will be selected to raise the sum rate.

If the judgment result is NO, the boundaries are adjusted based on the load radios of different sites.

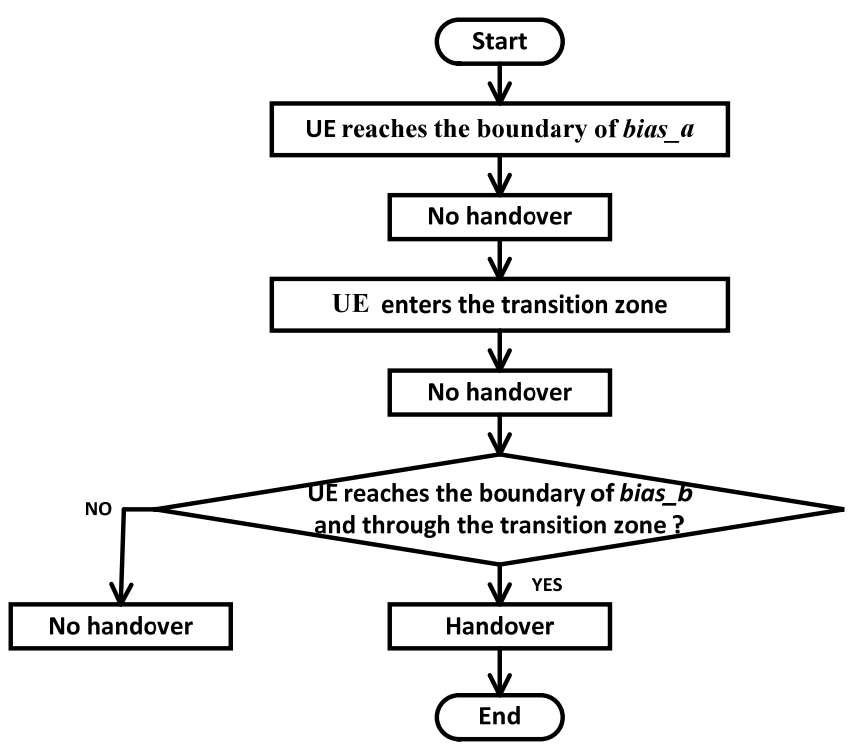

Figure 2 One example of serving cell selection

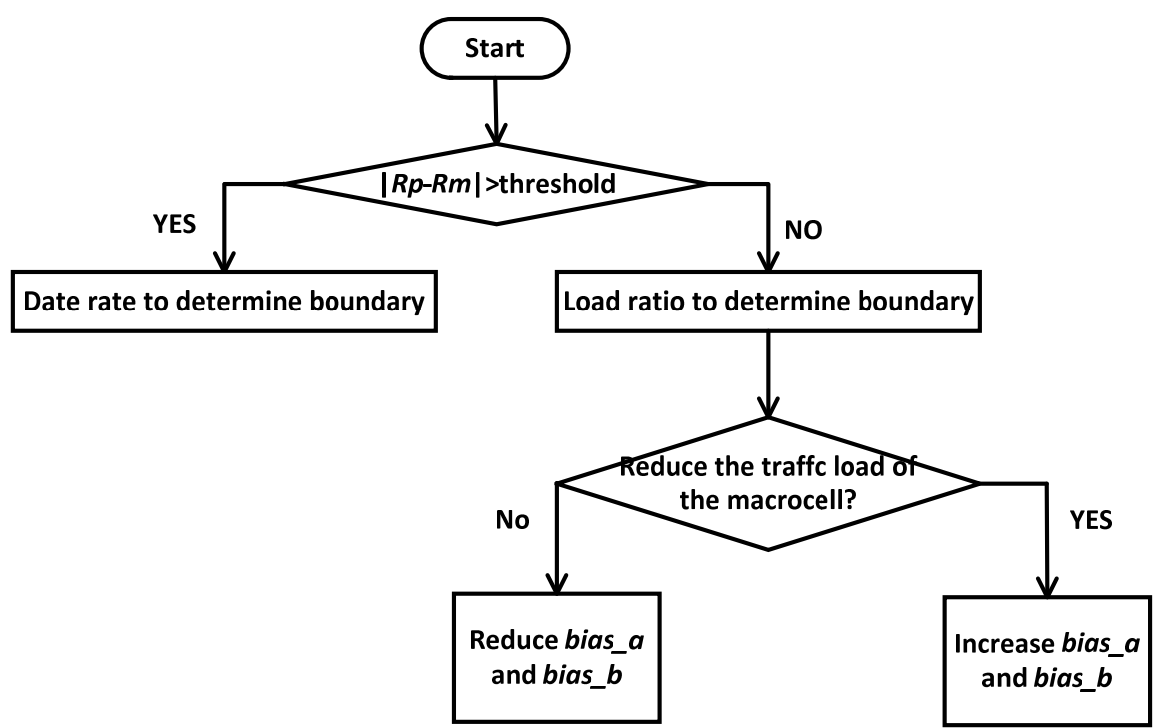

Figure 3 Changes in boundaries 


\section{Simulation Results}

In this section, simulation results for our proposed method compared with two methods are presented. In order to study the effect of transition zones in the proposed method conveniently, the bias cannot be adaptively adjusted in simulation. For method 1, traditional range expansion is employed, which is not adopted in method 2. The corresponding simulation parameters are listed in Table 1.

Table 1 Main simulation assumptions

\begin{tabular}{|c|c|}
\hline Parameter & Assumption \\
\hline \hline Inter-BS distance & $250[\mathrm{~m}]$ \\
\hline \hline $\begin{array}{c}\text { Number of Pico and Macro } \\
\text { Base Stations }\end{array}$ & 8,1 \\
\hline \hline Carrier Frequency & $2000[\mathrm{MHz}]$ \\
\hline \hline $\begin{array}{c}\text { Shadowing standard } \\
\text { deviation }\end{array}$ & $8[\mathrm{~dB}]$ \\
\hline Number of UEs & $\begin{array}{c}100 \text { UEs dropped in } \\
\text { the transition zone }\end{array}$ \\
\hline \hline UE Antenna pattern & Omni \\
\hline BS antenna gain & $5[\mathrm{dBi}]$ \\
\hline BS noise figure & $0[\mathrm{dBi}]$ \\
\hline \hline UE Antenna gain & $9[\mathrm{~dB}]$ \\
\hline UE Noise Figure & $30,46[\mathrm{dBm}]$ \\
\hline \hline Total Pico and Macro BS \\
TX power
\end{tabular}

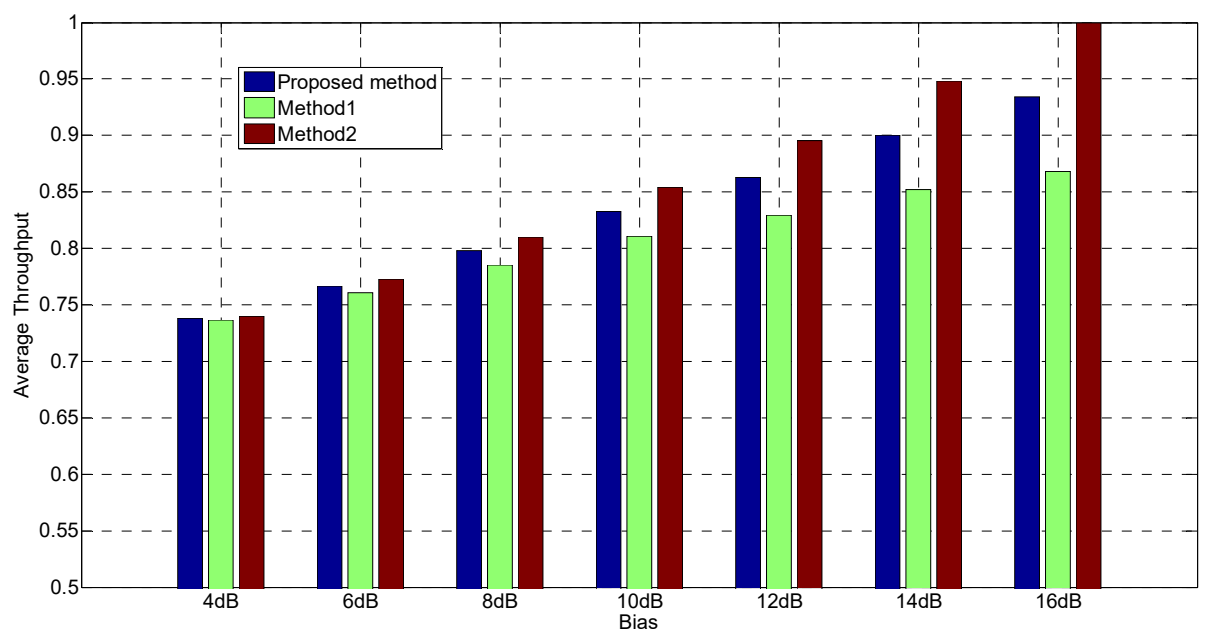

Figure 4 Average throughput versus bias 


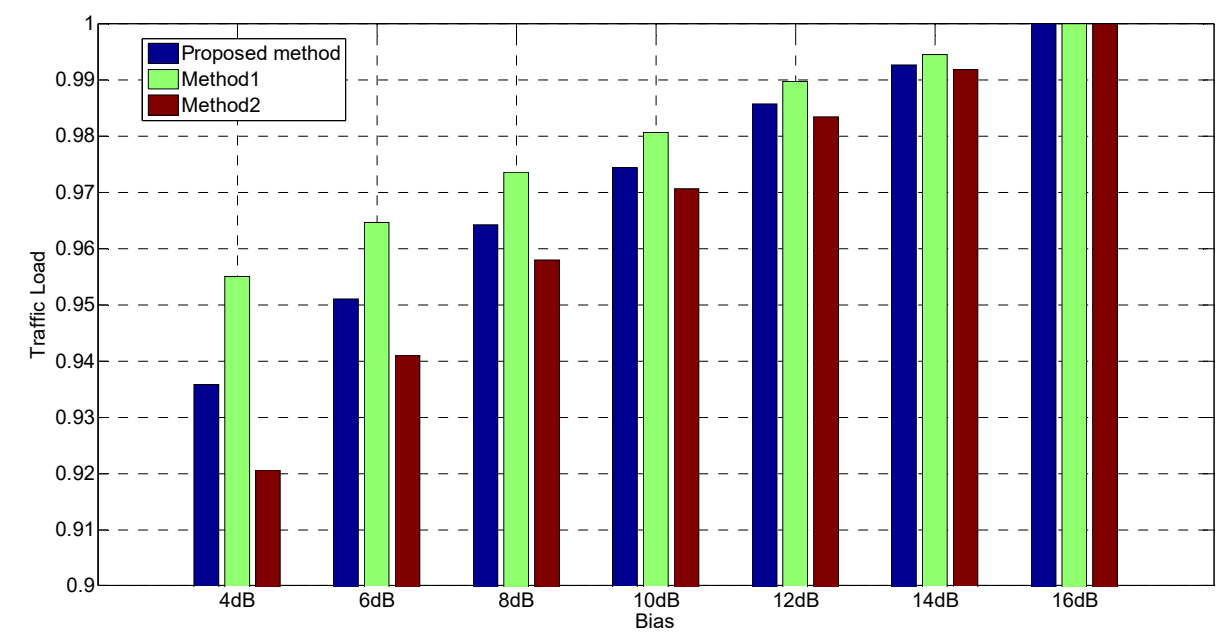

Figure 5 Traffic load versus bias

Figure 4 compares the normalized average throughput of all UEs versus bias_ $b$ in three methods in which the method 2 is chosen as the standard. Bias_a is set to $2 \mathrm{~dB}$. Since transmit powers are different between macro and pico sites, the throughput of method 2 is higher than the others. Our proposed method's performance is close to the best one while avoiding the ping-pong effect. Performance gap is not apparent when the bias is small, because the UEs in the transition zones are closer to macro BS with the increase of bias.

Figure 5 depicts the traffic load of three methods based on their maximum throughput under this scenario. The result clearly indicates that when the bias is low, the traffic load of method 1 is heavier than the others. And larger throughput range of method 2 causes load fluctuation more obviously.

\section{Conclusions}

In cellular networks with CRE, the ping-pong effect is considered to be undesirable for influencing the energy efficiency and the system reliability. In this paper, we proposed a new dynamic smooth handover method based on transition zones to avoid unnecessary cell handover. The results of simulation confirm the performance of our method with different transition zones. Furthermore, we are planning to study the reliable relations of the traffic load and the transition zones in fast moving scenes.

\section{Acknowledgements}

This research is supported by the University Natural Science Research Project of Anhui Province (KJ2017A004), the Startup Research Foundation of Anhui University (J01001958), the Open Project of Key Laboratory of Intelligent Computing and Signal Processing.

\section{References}

[1] Damnjanovic, A., Montojo, J., Wei, Y.B., Ji, T.F., Luo, T., Vajapeyam, M., Yoo, T., Song, O. and Malladi, D. (2011) A Survey on 3GPP Heterogeneous Networks, IEEE Wireless Communications, 18(3), 10-21.

[2] Lee, D., Seo, H., Clerckx. B., Hardouin, E., Mazzarese, D., Nagata, S. and Sayana K. (2012) Coordinated Multipoint Transmission and Reception in LTE-Advanced: Deployment Scenarios and Operational Challenges, IEEE Communications Magazine, 50(2), 148-155. 
[3] Oh, J. and Han, Y. (2012) Cell Selection for Range Expansion with Almost Blank Subframe in Heterogeneous Networks, 2012 IEEE 23rd International Symposium on Personal, Indoor and Mobile Radio Communications, 653-657.

[4] Yasir, B.A., Su, G. and Bachache, N. (2012) Range Expansion for Pico Cell in Heterogeneous LTE - A cellular Networks, 2012 2nd International Conference on Computer Science and Network Technology, 1235-1240.

[5] Güvenç, I., Jeong, M.-R., Demirdogen, I., Kecicioglu, B. and Watanabe, F. (2011) Range Expansion and Inter-Cell Interference Coordination (ICIC) for Picocell Networks, 1-6.

[6] Güvenç, I. (2011) Capacity and Fairness Analysis of Heterogeneous Networks with Range Expansion and Interference Coordination, IEEE Communications Letters, 15(10), 1084-1087.

[7] Gupta, R., Kalyanasundaram, S., Natarajan, B. and Sen, M. (2016) Performance Analysis of Enhanced Dynamic Point Selection CoMP Scheme for Heterogeneous Networks, 2016 IEEE 83rd Vehicular Technology Conference, 1-5.

[8] Michail, K., Tatsis, V., Panagiotis, D., Georgios, C., Nomikos, N., Skoutas, D.N., Vouyioukas, D. and Skianis, C. (2016) A Load and Channel Aware Dynamic Point Selection Algorithm for LTEA CoMP Networks, 2016 International Conference on Telecommunications and Multimedia, 1-5. 